\title{
Solutions for Klein-Gordon Equation in Randall-Sundrum-Kerr Scenario
}

\author{
Jéferson de Oliveira and Carlos Eduardo Pellicer de Oliveira \\ Instituto de Física, Universidade de São Paulo \\ C.P. 66318, 05315-970, São Paulo-SP, Brazil
}

(Received on 14 October, 2005)

\begin{abstract}
We study the scalar perturbations of rotating black holes in framework of extra dimensions type RandallSundrum(RS).
\end{abstract}

We study the scalar perturbations of a rotating black hole, in a framework of extra dimensions described by a RandallSundrum model[1]. The extraction of energy from rotating black holes is possible due to the well-known effect of superradiance[2-4]: the reflected wave has an amplitude larger than the incoming wave, and thereby is amplified. We consider the process of superradiance for this background. The conditions for superradiance and the reflection coefficients are found for scalar field perturbations.

We analyze the scalar perturbations of the metric

$$
d s^{2}=l^{2} / y^{2}\left[d s_{\text {Kerr }}^{2}+d y^{2}\right],
$$

in which $d s_{\text {Kerr }}^{2}$ is the Kerr metric in the Boyer-Lindquist coordiantes $(t, r, \theta, \phi)$.

The equation for scalar field dynamics $\Phi\left(X^{\mu}, y\right),\left(X^{\mu}=\right.$ $t, r, \theta, \phi)$ with mass $\mu^{2}$ is the Klein-Gordon equation

$$
\frac{1}{\sqrt{-g}} \frac{\partial}{\partial x^{A}}\left(g^{A B} \sqrt{-g} \frac{\partial \Phi}{\partial x^{B}}\right)+\mu^{2} \Phi=0 .
$$

Due to the axial symmetry and stationarity of the background metric we can expand the scalar field in the appropriate harmonics which are defined by the symetry group of motion of the metric, $\Phi\left(X^{\mu}, y\right)=R(r) S(\theta) \Omega(y) e^{i m \phi} e^{-i \omega t}$. After some algebra we obtain two equations, thereby decoupling the bulk evolution equation from the genuine brane fields. The angular and radial variables can be easily separated by the usual methods [6]. In the end, we obtain three equations with separated variables to radial, angular, and bulk coordinates,

$$
\begin{gathered}
\frac{d}{d r}\left(\Delta \frac{d R(r)}{d r}\right)+\frac{R(r)}{\Delta}\left[\left(r^{2}+a^{2}\right)^{2} \omega^{2}-4 \text { Mram } \omega+\right. \\
\left.+(m a)^{2}-\Delta\left((a \omega)^{2}+Q^{2} r^{2}+P\right)\right]=0, \\
\frac{1}{\sin \theta} \frac{d}{d \theta}\left(\sin \theta \frac{d S(\theta)}{d \theta}\right)+S(\theta)\left[\left(\omega^{2}-Q^{2}\right) a^{2} \cos ^{2} \theta\right] \\
-S(\theta)\left[\frac{m^{2}}{\sin ^{2} \theta}+P\right]=0, \\
\frac{d}{d y}\left(f(y)^{3 / 2} \frac{d \Omega(y)}{d y}\right)+Q^{2} \Omega(y) f(y)^{3 / 2}+\mu^{2} f(y)=0 .
\end{gathered}
$$

Let us make the usual replacements in order to reduce Eq.(3) to the standard wave-like type

$$
\frac{d^{2} \Psi(r)}{d r_{*}^{2}}+\Psi(r)\left[\omega^{2}-V(r, \omega)\right]=0,
$$

with the potential for massless scalar perturbation defined by

$$
\begin{gathered}
V(r, \omega)=\frac{4 M r a(m \omega)-(m a)^{2}+\Delta\left((a \omega)^{2}+Q^{2} r^{2}+P\right)}{\left(r^{2}+a^{2}\right)^{2}}+ \\
\frac{\Delta\left(3 r^{2}-4 M r+a^{2}\right)}{\left(r^{2}+a^{2}\right)^{3}}-\frac{3 \Delta^{2} r^{2}}{\left(r^{2}+a^{2}\right)^{4}}
\end{gathered}
$$

The branes are situated at $z=0$ and $z=d$ (or at $y=l$ and $y=l e^{d / l}$ respectively). Therefore, the solution for equation (5) is

$$
\Omega(y)=y^{2}\left[A J_{\sqrt{4-k^{2}}}(Q y)+B Y \sqrt{4-k^{2}}(Q y)\right],
$$

in which $A, B$ are constants, $J_{\sqrt{4-k^{2}}}(Q y), Y_{\sqrt{4-k^{2}}}(Q y)$ are Bessel's functions and $k^{2}=\mu^{2} l^{2}$.

In the two-brane world model, the boundary conditions for the perturbations comes from the Israel junction conditions [7] which imply the constraint for the massless scalar field

$$
Y_{1}\left(Q_{n}\right) J_{1}\left(x_{n}\right)=Y_{1}\left(x_{n}\right) J_{1}\left(Q_{n} l\right), \quad Q_{n}=\frac{x_{n}}{l} e^{l / d} .
$$

We note that at spatial infinity the "effective potential" $\omega^{2}-$ $V$ has the asymptotic form

$$
\omega^{2}-V \rightarrow \omega^{2}-Q^{2}, \quad r^{*} \rightarrow+\infty
$$

while at the event horizon the asymptotic form of the equation is

$$
\omega^{2}-V \rightarrow(\omega-m \Omega)^{2}, \quad r^{*} \rightarrow-\infty,
$$

in which $\Omega=\frac{a}{2 M r_{+}}, \quad r_{+}=M+\sqrt{M^{2}-a^{2}}$. In equation (10), we see that when $Q^{2}$ is larger than $\omega^{2}$ the effective potential is clearly negative at sufficiently large $r$ and the scalar field perturbations are unstable. Thus, we need to be restricted by the case when $Q^{2}<\omega_{q n}^{2}$, where $\omega_{q n}$ is the lowest (fundamental) quasinormal mode. In that case, the asymptotic solutions of the wave equation (6) have the form:

$$
\Psi \rightarrow B_{L m \omega} e^{-i(\omega-m \Omega) r^{*}}, \quad r^{*} \rightarrow-\infty
$$




$$
\Psi \rightarrow e^{-i\left(\sqrt{\omega^{2}-Q^{2}}\right) r^{*}}+A_{L m \omega} e^{i\left(\sqrt{\omega^{2}-Q^{2}}\right) r^{*}}, \quad r^{*} \rightarrow+\infty .
$$

Here the requirement that the wave should have an ingoing group velocity at the event horizon is satisfied. The wave comes from infinity, partially passes through the potential barrier reaching the event horizon, the rest reflects back. From the constancy of the Wronskian, i.e. from equality of the Wronskian at both asymptotics, we have: $1-\left|A_{L m \omega}\right|^{2}-$ $\left|B_{L m \omega}\right|^{2} \frac{\omega-m \Omega}{\sqrt{\omega^{2}-Q^{2}}}=0$. It means that, similar to the ordinary

Kerr case, $\left|A_{L m \omega}\right|>1$, i.e. the amplitude of the reflected wave is larger than that of the incident wave if the following condition of superradiance takes place:

$$
m \Omega>\omega .
$$

In order to find the reflection coefficient let us consider the near region wave behavior, when $r-r_{+} \ll 1 / \omega$. In this approximation Eq.(3) reads:

$$
\Delta \frac{d}{d r}\left(\Delta \frac{d R(r)}{d r}\right)+\left[r_{+}^{4}(\omega-m \Omega)^{2}-L(L+1) \Delta\right] R(r)=0 .
$$

The general solution of this equation is

$$
\begin{gathered}
R=A z^{-i \chi}(1-z)^{L+1} F(a-c+1, b-c+1,2-c, z)+ \\
B z^{i \chi}(1-z)^{L+1} F(a, b, c, z)
\end{gathered}
$$

in which

$$
\chi=(\omega-m \Omega) \frac{r_{+}^{2}}{r_{+}-r_{-}}, \quad z=\frac{r-r_{+}}{r-r_{-}} .
$$

Following [2] we obtain the reflection coefficient $b / a$

$$
\frac{b}{a}=2 i\left(\omega^{2}-Q^{2}\right)^{L+1 / 2} \chi \frac{(1-)^{L}}{2 L+1}\left(\frac{L !}{(2 L-1) !}\right)^{2}
$$

$$
\times \frac{\left(r_{+}-r_{-}\right)^{2 L+1}}{(2 L) !(2 L+1) !}\left(k^{2}+4 \chi^{2}\right) .
$$

By estimating the possible influence of the RS model onto superradiance let us find the eigenvalues $Q_{n}$ of the bulk equation (8). We can solve equation (9) numerically. The spectrum of egenvalues $Q_{n}$ does not depend on $d$, and depend on $l$ very mildy, provided that $l$ is small.

The first ten modes for $l=0.0001$ and $d=0.0005 \mathrm{~m}$. are shown in the Table I.

TABLE I: $Q_{n}$ eigenvalues

\begin{tabular}{|l|l||l|l|}
\hline$n$ & $Q_{n}$ & $n$ & $Q_{n}$ \\
\hline 1 & 3.83171 & 6 & 19.6159 \\
\hline 2 & 7.01559 & 7 & 22.7601 \\
\hline 3 & 10.1735 & 8 & 25.9037 \\
\hline 4 & 13.3237 & 9 & 29.0468 \\
\hline 5 & 16.4706 & 10 & 32.1897 \\
\hline
\end{tabular}

For black holes of one tenth of solar mass we definitely avoid GL instability [8]. In spite of this, the superradiant instability hinted by [9] appears, because this massless scalar perturbation simulate a massive parturbation in the 4-dimensional Kerr black hole which is unstable.

\section{Acknowledgments}

We wish to acknowledge useful discussions with Roman Konoplya, Karlucio H. Castello-Branco and Prof. Elcio Abdalla. This work is supported by FAPESP.
[1] L. Randall and R.Sundrum, Phys. Rev. Lett. 83, 3370 (1999).

[2] A. A. Starobinsky, Zh. Eksp. Teor. Fiz. 64, 48 (1973) [Sov. Phys. JETP 37, 28 (1973)].

[3] A. A. Starobinsky and S. M. Churilov, Zh. Eksp. Teor. Fiz. 65, 3 (1973) [Sov. Phys. JETP 38, 1 (1973)].

[4] Ya. B. Zel'dovich Pis'ma, Zh. Eksp. Teor. Fiz. 14, 270 (1971) [JETP Lett 14, 180 (1971)]; Ya. B. Zel'dovich, Zh. Eksp. Teor. Fiz. 62, 2076 (1972) [Sov. Phys. JETP 35, 1085 (1972)].

[5] M. S. Modgil, S. Panda and G. Sengupta, Mod.Phys. Lett. A17, 1479 (2002).
[6] K. D. Kokkotas and B. G. Schmidt, Liv. Rev. Rel. 2, 2 (1999).

[7] W. Israel, Nuovo Cim. B 44S10 (1966) 1 [Erratum-ibid. B 48 (1967 NUCIA,B44,1.1966) 463]; R. Maartens, Liv. Rev. Rel. 7, 7 (2004); E. Abdalla, A. Casali and B. Cuadros-Melgar, Nucl. Phys B 44, 201 (2002), Int. Theor. Phys 43, 801 (2004).

[8] R. Gregory and R. Laflamme, Phys. Rev. Lett. 70, 2837 (1993).

[9] D. Marolf and B.C. Palmer, Phys. Rev D 70, 084045 (2004); V. Cardoso and S. Yoshida, JHEP 0507, 009 (2005). 